DOI:10.22337/2587-9618-2018-14-०-००-८०

\title{
NUMERICAL ANALYSIS OF NON-LINEAR VIBRATIONS OF A FRACTIONALLY DAMPED CYLINDRICAL SHELL UNDER THE ADDITIVE COMBINATIONAL INTERNAL RESONANCE
}

\author{
Basem Ajarmah $^{1,2}$, Marina V. Shitikova ${ }^{1}$ \\ ${ }^{1}$ Voronezh State Technical University, Voronezh, RUSSIA \\ ${ }^{2}$ Al-istiqlal University, Jericho, PALESTINE
}

\begin{abstract}
Non-linear damped vibrations of a cylindrical shell subjected to the additive type combinational internal resonance are investigated numerically using two different numerical methods. The damping features of the surrounding medium are described by the fractional derivative Kelvin-Voigt model involving the RiemannLiouville fractional derivatives. Within the first method, the generalized displacements of a coupled set of nonlinear ordinary differential are estimated using numerical solution of nonlinear multi-term fractional differential equations by the procedure based on the reduction of the problem to a system of fractional differential equations. According to the second method, the amplitudes and phases of nonlinear vibrations are estimated from the governing nonlinear differential equations describing amplitude-and-phase modulations for the case of the additive combinational internal resonance. A good agreement in results is declared.
\end{abstract}

Keywords: cylindrical shell, free nonlinear damped vibrations, additive combinational internal resonance, method of multiple time scales, multi-term fractional differential equations

\section{ЧИСЛЕННЫЙ АНАЛИЗ НЕЛИНЕЙНЫХ КОЛЕБАНИЙ ЦИЛИНДРИЧЕСКОЙ ОБОЛОЧКИ С ДРОБНЫМ ДЕМПФИРОВАНИЕМ ПРИ АДДИТИВНОМ КОМБИНАЦИОННОМ ВНУТРЕННЕМ РЕЗОНАНСЕ}

\author{
Б. Айармах ${ }^{1,2}$, М.В. Шитикова ${ }^{1}$ \\ ${ }^{1}$ Воронежский государственный технический университет, г. Воронеж, РОССИЯ \\ ${ }^{2}$ Al-istiqlal University, г. Иерихон, ПАЛЕСТИНА
}

\begin{abstract}
Аннотация: Рассматриваются нелинейные затухающие колебания цилиндрической оболочки при аддитивном комбинационном внутреннем резонансе. Для решения соответствующих задач применяются два различных численных метода. Демпфирующие особенности окружающей среды описываются с помощью дробной производной модели Кельвина-Фойгта, включающей дробные производные РиманаЛиувилля. В рамках первого метода обобщенные смещения связного набора нелинейных обыкновенных дифференциалов оцениваются на основе численного решения нелинейных многочленных уравнений с дробными производными по методике, предусматривающей сведение исходной задачи к системе уравнений с дробными производными. Согласно второму методу, амплитуды и фазы нелинейных колебаний оцениваются из определяющих нелинейных дифференциальных уравнений, описывающих амплитудно-фазовые модуляции для случая аддитивного комбинационного внутреннего резонанса. Отмечена хорошая согласованность полученных результатов.
\end{abstract}

Ключевые слова: цилиндрическая оболочка, свободные нелинейно-затухающие колебания, аддитивный комбинационный внутренний резонанс, метод кратных временных шкал, многочленные уравнения с дробными производными 
Numerical Analysis of Non-linear Vibrations of a Fractionally Damped Cylindrical Shell under the Additive Combinational Internal Resonance

\section{INTRODUCTION}

In mechanical nonlinear vibrations, the phenomena of internal resonance and energy exchange are quite often what requires the thorough studies, since in the case of low damping it could result in long-time vibrations accompanied by the two-sided or one-sided energy interchange between coupled modes [1]. It will suffice to mention the state-of-the-art articles [1,2] and the monograph [3] involving the extensive review of literature in the field of internal resonances in different mechanical systems. Different types of the internal resonance: one-to-one, two-to-one, three-to-one, as well as a variety of combinational resonances, when three and more natural modes interact, have been discussed. The enumerated internal resonances were investigated in various mechanical systems with multiple degree-offreedom, as well as in strings, beams, plates, and shells.

It has been emphasized by many researchers [413] that the phenomenon of internal resonances can be very critical especially for circular cylindrical shells. Thus, the nonlinear vibrations of infinitely long circular cylindrical shells under the conditions of the two-to-one internal resonance were studied in [6] via the method of multiple time scales using the simple plane strain theory of shells. Parametrically excited vibrations of infinitely long cylindrical shells and nonlinear forced vibrations of a simply supported, circular cylindrical shell filled with an incompressible, inviscid, quiescent and dense fluid were investigated in $[4,5,7]$ using Donnell's nonlinear shallow-shell theory. The flexural deformation is usually expanded by using the linear shell eigenmodes, in so doing the flexural response involves several nodal diameters and one or two longitudinal halfwaves. Internal resonances of different types have been analyzed in [8-13].

The extensive review of studies on shallow shells nonlinear vibrations could be found in the state-of-the-art articles [14-16]. In spite of the fact that many studies have been carried out on large amplitude vibrations of circular cylindrical shells and many different approaches to the problem have been used, we agree with Breslavsky and Amabili [10] that this research area is still far from being well understood.

In recent years much attention is given to damping features of mechanical systems subjected to the conditions of different internal resonances. Damping properties of nonlinear systems are described mainly by the first-order time-derivative of a generalized displacement [3]. However, as it has been shown by Rossikhin and Shitikova [17], who analyzed free damped vibrations of suspension combined system under the conditions of the one-to-one internal resonance, for good fit of the theoretical investigations with the experimental results it is better to describe the damping features of nonlinear mechanical systems in terms of fractional time-derivatives of the generalized displacements [18].

During the last decade, fractional calculus entered the mainstream of engineering analysis. And it has been widely applied to structural dynamics problems both in discrete and continuous equations. The history of the fractional calculus applications in mechanics could be found in the retrospective paper by Rossikhin [19], while a comprehensive review of the fractional calculus models in different dynamic problems of solids and structures is presented in the state-of-the-art article [18], wherein the results obtained in the field critically estimated in the light of the present view of the place and role of the fractional calculus in engineering problems and practice.

It has been suggested in 2011 to examine the nonlinear dynamic response of a thin cylindrical shell vibrating in a fractionally damped medium [20], when the dynamic behavior of the shell is described by a set of three coupled nonlinear differential equations with due account for the fact that the shell is being under the conditions of the internal resonance resulting in the interaction of modes corresponding to the mutually orthogonal displacements. The 
displacement functions are determined in terms of eigenfunctions of linear vibrations.

A new procedure resulting in decoupling linear parts of equations has been proposed in Rossikhin and Shitikova [21] with the further utilization of the method of multiple scales for solving nonlinear governing equations of motion, in so doing the amplitude functions are expanded into power series in terms of the small parameter and depend on different time scales. It is shown that the phenomenon of the internal resonance between vibrational subsystems of the cylindrical shell under consideration can be very critical, since in the circular cylindrical shell of such a type the two-to-one [21], one-toone, three-to-one [22] internal resonances, as well as combinational internal resonances [23] could occur, which are governed by the order of smallness of viscosity. All possible cases of the internal resonance have been recently revealed in [22], which belong to the resonances of the constructive type, since all of them depend on the geometrical dimensions of the shell under consideration and its mechanical characteristics, that is why such resonances could not be ignored and eliminated for a particularly designed shell. It has been shown that the energy exchange could occur between two or three subsystems at a time: normal vibrations of the shell, its torsional vibrations and shear vibrations along the shell axis. Such an energy exchange, if it takes place for a rather long time, could result in crack formation in the shell, and finally to its failure. The energy exchange has been illustrated pictorially by the phase portraits, wherein the phase trajectories of the phase fluid motion are depicted.

In the present paper, we are going to verify parameter values of the cylindrical shell model [20-23], resulting in the nonlinear vibrations of a fractionally damped cylindrical shell under the conditions of combinational internal resonance, and to study such phenomenon using two different numerical methods [24]. In the first method, the generalized displacements of a coupled set of nonlinear ordinary differential equations of the second order are estimated using numerical solution of nonlinear multiterm fractional differential equations by the procedure based on the reducing of the problem to a system of fractional differential equations [25-28]. According to the second method, the amplitudes and phases of nonlinear vibrations are estimated from the governing nonlinear differential equations describing amplitude-andphase modulations for the case of the combinational internal resonance [23] using the Runge-Kutta fourth order method.

\section{PROBLEM FORMULATION AND GOVERNING EQUATIONS}

Let us examine the dynamic response of a free supported non-linear elastic circular cylindrical shell of radius $R$ and length $l$, vibrations of which in the cylindrical system of coordinates described by the Donnell-Mushtari-Vlasov equations with respect to the three displacements [12] considering that damping features of the surrounding medium are described by the time-differential operator of the fractional order [20]:

$$
\begin{aligned}
& \frac{\partial^{2} u}{\partial x^{2}}+\frac{1-\sigma}{2} \frac{1}{R^{2}} \frac{\partial^{2} u}{\partial \varphi^{2}}+ \\
& +\frac{1+\sigma}{2} \frac{1}{R} \frac{\partial^{2} v}{\partial x \partial \varphi}-\sigma \frac{1}{R} \frac{\partial w}{\partial x}+\frac{\partial w}{\partial x} \frac{\partial^{2} w}{\partial x^{2}}+ \\
& +\frac{1+\sigma}{2} \frac{1}{R^{2}} \frac{\partial w}{\partial \varphi} \frac{\partial^{2} w}{\partial x \partial \varphi}+\frac{1-\sigma}{2} \frac{1}{R^{2}} \frac{\partial w}{\partial x} \frac{\partial^{2} w}{\partial \varphi^{2}}= \\
& =\frac{\rho\left(1-\sigma^{2}\right)}{E} \frac{\partial^{2} u}{\partial t^{2}}+\alpha_{1}\left(\frac{d}{d t}\right)^{\gamma} u, \\
& \quad \frac{1}{R^{2}} \frac{\partial^{2} v}{\partial \varphi^{2}}+\frac{1-\sigma}{2} \frac{\partial^{2} v}{\partial x^{2}}+\frac{1+\sigma}{2} \frac{1}{R} \frac{\partial^{2} u}{\partial x \partial \varphi}- \\
& -\frac{1}{R^{2}} \frac{\partial w}{\partial \varphi}+\frac{1}{R^{3}} \frac{\partial w}{\partial \varphi} \frac{\partial^{2} w}{\partial \varphi^{2}}+ \\
& +\frac{1+\sigma}{2} \frac{1}{R} \frac{\partial w}{\partial x} \frac{\partial^{2} w}{\partial x \partial \varphi}+\frac{1-\sigma}{2} \frac{1}{R} \frac{\partial w}{\partial \varphi} \frac{\partial^{2} w}{\partial x^{2}}= \\
& =\frac{\rho\left(1-\sigma^{2}\right)}{E} \frac{\partial^{2} v}{\partial t^{2}}+a e_{2}\left(\frac{d}{d t}\right)^{\gamma} v,
\end{aligned}
$$


Numerical Analysis of Non-linear Vibrations of a Fractionally Damped Cylindrical Shell under the Additive Combinational Internal Resonance

$$
\begin{aligned}
& \frac{h^{2}}{12} \nabla^{4} w+\frac{1}{R^{2}} w-\sigma \frac{1}{R} \frac{\partial u}{\partial x}-\frac{1}{R^{2}} \frac{\partial v}{\partial \varphi}- \\
& -\frac{1}{2} \frac{\sigma}{R}\left(\frac{\partial w}{\partial x}\right)^{2}-\frac{1}{2} \frac{1}{R^{3}}\left(\frac{\partial w}{\partial \varphi}\right)^{2}- \\
& -\frac{\partial}{\partial x}\left[\frac{\partial w}{\partial x}\left(\frac{\partial u}{\partial x}+\frac{\sigma}{R} \frac{\partial v}{\partial \varphi}-\frac{\sigma}{R} w\right)+\right. \\
& \left.+\frac{1-\sigma}{2} \frac{1}{R} \frac{\partial w}{\partial \varphi}\left(\frac{1}{R} \frac{\partial u}{\partial \varphi}+\frac{\partial v}{\partial x}\right)\right]- \\
& -\frac{1}{R} \frac{\partial}{\partial \varphi}\left[\frac{1}{R} \frac{\partial w}{\partial \varphi}\left(\sigma \frac{\partial u}{\partial x}+\frac{1}{R} \frac{\partial v}{\partial \varphi}-\frac{1}{R} w\right)+\right. \\
& \left.+\frac{1-\sigma}{2} \frac{\partial w}{\partial x}\left(\frac{1}{R} \frac{\partial u}{\partial \varphi}+\frac{\partial v}{\partial x}\right)\right]- \\
& =-\frac{\rho\left(1-\sigma^{2}\right)}{E} \frac{\partial^{2} w}{\partial t^{2}}-a_{3}\left(\frac{d}{d t}\right)^{\gamma} w
\end{aligned}
$$

where $x$-axis is directed along the axis of the cylinder, $\varphi$ is the polar angle in the plane perpendicular to the $x$-axis,

$$
u=u(x, \varphi, t), v=v(x, \varphi, t), \text { and } w=w(x, \varphi, t)
$$

are the displacements of points located in the shell's middle surface in three mutually orthogonal directions $x, \varphi, r$ with $r$ as the polar radius, $h$ is the thickness, $\rho$ is the density, $E$ and $\sigma$ are the elastic modulus and Poisson's ratio, respectively, $t$ is the time, $\alpha_{1}, \alpha_{2}, \alpha_{3}$ are the damping coefficients, and

$$
\nabla^{4}=\nabla^{2} \nabla^{2}=\frac{\partial^{4}}{\partial x^{4}}+2 \frac{1}{R^{2}} \frac{\partial^{4}}{\partial x^{2} \partial \varphi^{2}}+\frac{1}{R^{4}} \frac{\partial^{4}}{\partial \varphi^{4}}
$$

The initial conditions

$$
\begin{gathered}
\left.u\right|_{t=0}=\left.v\right|_{t=0}=\left.w\right|_{t=0} ; \\
\left.\dot{u}\right|_{t=0}=\varepsilon V_{1}^{0}(x, \varphi), \\
\left.\dot{v}\right|_{t=0}=\varepsilon V_{2}^{0}(x, \varphi), \\
\left.\dot{w}\right|_{t=0}=\varepsilon V_{3}^{0}(x, \varphi)
\end{gathered}
$$

where $V_{i}^{0}(x, \varphi) \quad(i=1,2,3)$ are the corresponding initial velocities, and $\varepsilon$ is a small value, should be added to Eqs. (1)-(3). Hereafter over dots denote time-derivatives.

The boundary conditions for the simply supported shell (the Navier-type conditions for the edges free supported in the $x$-direction) have the form [12]:

$$
\begin{gathered}
\left.w\right|_{x=0}=\left.w\right|_{x=l}=0,\left.\quad v\right|_{x=0}=\left.v\right|_{x=l}=0, \\
\left.\frac{\partial^{2} w}{\partial x^{2}}\right|_{x=0}=\left.\frac{\partial^{2} w}{\partial x^{2}}\right|_{x=l}=0, \\
\left.\frac{\partial u}{\partial x}\right|_{x=0}=\left.\frac{\partial u}{\partial x}\right|_{x=l}=0 .
\end{gathered}
$$

From relationships (5) it follows that free vibrations are excited by the weak disturbance from the equilibrium position.

It has been proposed in [20] to rewrite Eqs. (1)(5) in the nondimensioned form in terms of the following dimensionless parameters:

$$
\begin{array}{ll}
u^{*}=\frac{u}{l}, & v^{*}=\frac{v}{l}, \quad w^{*}=\frac{w}{l}, \\
x^{*}=\frac{x}{l}, & t^{*}=\frac{t}{l} \sqrt{\frac{E}{\rho\left(1-\sigma^{2}\right)} .}
\end{array}
$$

Dropping hereafter the asterisks for the ease of presentation, let us admit the solution of the Navier type for Eqs. (1)-(3) in the form

$$
\begin{aligned}
& u(x, \varphi, t)=\sum_{m=1}^{\infty} \sum_{n=1}^{\infty} x_{1 m n}(t) \eta_{1 m n}(x, \varphi) ; \\
& v(x, \varphi, t)=\sum_{m=1}^{\infty} \sum_{n=1}^{\infty} x_{2 m n}(t) \eta_{2 m n}(x, \varphi) ; \\
& w(x, \varphi, t)=\sum_{m=1}^{\infty} \sum_{n=1}^{\infty} x_{3 m n}(t) \eta_{3 m n}(x, \varphi),
\end{aligned}
$$

where $x_{j m n}(t)$ and $\eta_{j m n}(x, \varphi) \quad(j=1,2,3)$ are, respectively, the generalized displacements and eigenfunctions satisfying the boundary conditions (6), and $m$ and $n$ are integers. 
Distinct to the traditional modeling the viscous resistance forces via first order time-derivatives [16], in the present research we adopt the fractional order time-derivative

$$
(d / d t)^{\gamma}
$$

what, as it had been shown in [17,18], allows one to obtain the damping coefficients dependent on the natural frequency of vibrations. It has been demonstrated in [29] on the example of the Golden Gate suspension bridge that such an approach for modeling the damped non-linear vibrations provides the good agreement between the theoretical results and the experimental data through the appropriate choice of the fractional parameter (the order of the fractional derivative) and the viscosity coefficient.

It was shown in Samko et al. [30] (see Chapter 2, Paragraph 5, point $7^{0}$ ) that the fractional order of the operator of differentiation

\section{$(d / d t)^{\gamma}$}

is equal to the Marsho fractional derivative, which, in its turn, equal to the RiemannLiouville derivative $D_{+}^{\gamma}$.

It has been noted in $[17,18]$ that a fractional derivative is the immediate extension of an ordinary derivative. In fact, when $\gamma \rightarrow 1$ the fractional derivative goes over into the ordinary time-derivative of the first order, and the mathematical model of the viscoelastic shell under consideration transforms into the conventional Kelvin-Voigt model, wherein the elastic element behaves non-lineally, but the viscous element behaves linearly. When

$$
\gamma \rightarrow 0
$$

the fractional derivative

$$
D_{+}^{\gamma} f \text { tends to } f(t) \text {. }
$$

To put it otherwise, the introduction of the new fractional parameter along with the parameters $a_{i}$ allows one to change not only the magnitude of viscosity at the cost of an increase or decrease in the parameters $c_{i}$, but also the character of viscosity at the sacrifice of variations in the fractional parameter $\gamma$.

Now substituting the proposed solution (7) in nondimensioned Eqs. (1)-(3), multiplying then each equation by the corresponding function $\eta_{j m n}(x, \varphi)$, integrating over $x$ and $\varphi$, and using the orthogonality conditions for linear modes within the domains of

$$
0 \leq x \leq 1 \text { and } 0 \leq \varphi \leq 2 \pi,
$$

we are led to a coupled set of nonlinear ordinary differential equations of the second order in $x_{i m n}(t)$. However, a new procedure has been proposed in [21] for decoupling the linear parts of nonlinear differential equations.

Thus, the system is reduced to the following form:

$$
\begin{aligned}
& \ddot{X}_{1 m n}+\alpha_{1} D^{\gamma} X_{1 m n}+\Omega_{1 m n}^{2} X_{1 m n}= \\
& =-\sum_{i=1}^{3} F_{i m n} L_{i m n}^{I} ; \\
& \ddot{X}_{2 m n}+\alpha_{2} D^{\gamma} X_{2 m n}+\Omega_{2 m n}^{2} X_{2 m n}= \\
& =-\sum_{i=1}^{3} F_{i m n} L_{i m n}^{I I} ; \\
& \ddot{X}_{3 m n}+\alpha_{3} D^{\gamma} X_{3 m n}+\Omega_{3 m n}^{2} X_{2 m n}= \\
& =-\sum_{i=1}^{3} F_{i m n} L_{i m n}^{I I I},
\end{aligned}
$$

where $D^{\gamma}=(d / d t)^{\gamma}$, and $X_{i}(i=1,2,3)$ are new generalized displacements which are connected with $x_{i m n}(t)$ via eigenvectors $L_{i m n}^{I}, L_{i m n}^{I I}, L_{i m n}^{I I I}$

$$
x_{i m n}(t)=X_{1 m n} L_{i m n}^{I}+X_{2 m n} L_{i m n}^{I I}+X_{3 m n} L_{i m n}^{I I I}
$$

of the matrix $S_{i j}^{m n}$ with the corresponding eigenvalues $\Omega_{1 \mathrm{mn}}, \Omega_{2 \mathrm{mn}}$, and $\Omega_{3 \mathrm{mn}}$, the elements of which are the following: 
Numerical Analysis of Non-linear Vibrations of a Fractionally Damped Cylindrical Shell under the Additive Combinational Internal Resonance

$$
S_{i j}^{m n}=\left[\begin{array}{ccc}
S_{11}^{m n} & S_{12}^{m n} & S_{13}^{m n} \\
S_{21}^{m n} & S_{22}^{m n} & S_{23}^{m n} \\
S_{31}^{m n} & S_{32}^{m n} & S_{33}^{m n}
\end{array}\right]=\left[\begin{array}{ccc}
\left(\pi^{2} m^{2}+\frac{1-\sigma}{2} \beta_{1}^{2} n^{2}\right) & \frac{1+\sigma}{2} \beta_{1} \pi m n & \sigma \beta_{1} \pi m \\
\frac{1+\sigma}{2} \beta_{1} \pi m n & \left(\frac{1-\sigma}{2} \pi^{2} m^{2}+\beta_{1}^{2} n^{2}\right) & \beta_{1}^{2} n \\
\sigma \beta_{1} \pi m & \beta_{1}^{2} n & \frac{\beta_{2}^{2}}{12}\left(\pi^{2} m^{2}+\beta_{1}^{2} n^{2}\right)^{2}+\beta_{1}
\end{array}\right],
$$

where

$$
\beta_{1}=l / R \text { and } \beta_{2}=h / l
$$

weakly varying with time could be represented by a uniform expansion in terms of different time scales:

$X_{i}=\varepsilon X_{i 1}\left(T_{0}, T_{1}, \ldots\right)+\varepsilon^{2} X_{i 2}\left(T_{0}, T_{1}, \ldots\right)+\ldots$

the shell.

From Eqs. (8)-(10) it is seen that their left-hand side parts are linear and independent of each other, while they are coupled only by non-linear terms $F_{i m n}$ in their right-hand sides.

It is known [3, 31] that during nonstationary excitation of thin bodies not all possible modes of vibration would be excited. Moreover, the modes which are strongly coupled by any of the so-called internal resonance conditions are initiated and dominate in the process of vibration, resulting in the energy transfer from one subsystem to another between the coupled modes, in so doing the types of modes to be excited are dependent of the character of the external excitation. It was emphasized in [31] that in the presence of damping, all modes that are not directly or indirectly excited by an internal resonance decay with time.

Assume hereafter that the vibration process occurs in such a way that only three natural modes corresponding to the complex generalized displacements

$$
X_{1 s_{1} s_{2}}, X_{2 l_{1} l_{2}} \text {, and } X_{3 k_{1} k_{2}}
$$

are excited and dominate over other natural modes. In this case, the right parts of Eqs. (8)(10) are significantly simplified.

According to [20], the approximate solution of these three nonlinear equations (wherein the low indices $s_{1} s_{2}, l_{1} l_{2}$ and $k_{1} k_{2}$ are omitted for the ease of presentation) for small but finite amplitudes

where $i=1,2,3, \varepsilon$ is a small dimensionless parameter of the same order of magnitude as the amplitudes,

$$
T_{n}=\varepsilon^{2} t \quad(n=0,1,2, \ldots)
$$

are new independent variables, among them:

$$
T_{0}=t
$$

is a fast scale characterizing motions with the natural frequencies, and

$$
T_{1}=\varepsilon t
$$

is a slow scale characterizing the modulation of the amplitudes and phases of the modes with nonlinearity.

Applying the method of multiple scales directly to the governing partial-differential equations by substituting (12) in them and considering that the first and second timederivatives, as well as the fractional order timederivative are defined in terms of new time scales, respectively, as follows:

$$
\frac{d}{d t}=D_{0}+\varepsilon D_{1}+\ldots, \quad \frac{d^{2}}{d t^{2}}=D_{0}^{2}+2 \varepsilon D_{0} D_{1} \ldots
$$




$$
\begin{aligned}
& \left(\frac{d}{d t}\right)^{\gamma}=D^{\gamma}=\left(D_{0}+\varepsilon D_{1}+\ldots\right)^{\gamma}= \\
& =D_{0}^{\gamma}+\varepsilon \gamma D_{0}^{\gamma-1} D_{1}+\frac{1}{2} \varepsilon^{2} \gamma(\gamma-1) D_{0}^{\gamma-2} D_{1}^{2}+\ldots
\end{aligned}
$$

where $D_{n}=\partial / \partial T_{n}$, and $D_{0}^{\gamma}, D_{0}^{\gamma-1}, D_{0}^{\gamma-2} \ldots$ are the Riemann-Liouville fractional derivatives in time $t[17,18]$, after separating the terms at the same powers of $\varepsilon$, we could obtain the equations corresponding to different orders of $\varepsilon$ [21].

It should be noted that the expansion of the fractional order operator of differentiation (14) for the first time was suggested in 1998 by Rossikhin and Shitikova [17], and nowadays it is used by the researchers worldwide when solving the nonlinear dynamic problems with fractional order damping.

The case of the order of $\varepsilon$ has been considered in detail in [21], wherein all types of the internal resonance, which could occur on this step, have been detected and classified: (1) the two-to-one internal resonance, when one natural frequency is twice the other natural frequency, (2) the oneto-one-to-two or one-to-two-to-two internal resonance, and (3) the combinational resonances of the additive-difference type of the first order, among them, the case of $\Omega_{2}=\Omega_{1}+\Omega_{3}$, which we are going to study below numerically.

Utilizing the procedure described in [22] and considering that the fractional order damping coefficients have the form of

$$
\alpha_{i}=\varepsilon \mu_{i} \tau_{i}^{\gamma}
$$

where $\tau_{i}$ is the relaxation time of the $i$-th generalized displacement and $\mu_{i}$ is a finite value, the following six first-order nonlinear ordinary-differential equations governing the modulation of the amplitudes and phases of the three interacting modes in case of combinational additive internal resonance $\Omega_{2}=\Omega_{1}+\Omega_{3}$ have been obtained:

$$
\begin{gathered}
\left(a_{1}^{2}\right)^{\cdot}+s_{1} a_{1}^{2}=-\Omega_{1}^{-1} a_{23}^{I} a_{1} a_{2} a_{3} \sin \delta ; \\
\left(a_{2}^{2}\right)^{\cdot}+s_{2} a_{2}^{2}=\Omega_{2}^{-1} a_{13}^{I I} a_{1} a_{2} a_{3} \sin \delta ; \\
\left(a_{3}^{2}\right)^{\cdot}+s_{3} a_{3}^{2}=-\Omega_{3}^{-1} a_{12}^{I I I} a_{1} a_{2} a_{3} \sin \delta ; \\
\dot{\varphi}_{1}-\frac{1}{2} \sigma_{1}-\frac{1}{2} \frac{a_{23}^{I}}{\Omega_{1}} \frac{a_{2} a_{3}}{a_{1}} \cos \delta=0 ; \\
\dot{\varphi}_{2}-\frac{1}{2} \sigma_{2}-\frac{1}{2} \frac{a_{13}^{I I}}{\Omega_{2}} \frac{a_{1} a_{3}}{a_{2}} \cos \delta=0 ; \\
\dot{\varphi}_{3}-\frac{1}{2} \sigma_{3}-\frac{1}{2} \frac{a_{12}^{I I I}}{\Omega_{3}} \frac{a_{1} a_{2}}{a_{3}} \cos \delta=0 ;
\end{gathered}
$$

where $a_{i}$ and $\varphi_{i}(i=1,2,3)$ are the amplitudes and phases, respectively,

$$
\delta=\varphi_{2}-\left(\varphi_{1}+\varphi_{3}\right)
$$

is the phase difference, an over dot denotes the differentiation with respect to $T_{1}$,

$$
\begin{gathered}
s_{i}=\mu_{i} \tau_{i}^{\gamma} \Omega_{i}^{\gamma-1} \sin \psi, \quad \sigma_{i}=\mu_{i} \tau_{i}^{\gamma} \Omega_{i}^{\gamma-1} \cos \psi(i=1,2,3), \\
\psi=\frac{1}{2} \pi \gamma,
\end{gathered}
$$

and $a_{23}^{\mathrm{I}}, a_{13}^{\mathrm{II}}, a_{12}^{\mathrm{III}}$ are constant coefficients defined by the coupled modes of vibrations [22].

\section{NUMERICAL METHOD OF SOLUTION}

\subsection{Defining the shell parameters that satisfy the condition of the combinational internal resonance $\Omega_{2}=\Omega_{1}+\Omega_{3}$}

Before proceeding to numerical investigations, let us find the shell parameters which could satisfy the condition of the additive combinational internal resonance

$$
\Omega_{2}=\Omega_{1}+\Omega_{3} .
$$


Numerical Analysis of Non-linear Vibrations of a Fractionally Damped Cylindrical Shell under the Additive Combinational Internal Resonance

Table 1. Part of shell parameters which satisfy the resonance condition $\Omega_{2}=\Omega_{1}+\Omega_{3}$.

\begin{tabular}{|c|c|c||c|c|c||c|c|c||c|c|c|}
\hline $\mathbf{\Omega}_{\mathbf{1}}$ & $m_{1}$ & $n_{1}$ & $\mathbf{\Omega}_{\mathbf{2}}$ & $m_{2}$ & $n_{2}$ & $\mathbf{\Omega}_{\mathbf{3}}$ & $m_{3}$ & $n_{3}$ & $\sigma$ & $\beta_{1}$ & $\beta_{2}$ \\
\hline 30.4137 & 5 & 3 & 44.412 & 4 & 5 & 13.9983 & 3 & 1 & 0.33 & 8.37 & 0.004 \\
\hline 27.0251 & 5 & 2 & 43.1784 & 3 & 4 & 16.1531 & 3 & 1 & 0.33 & 10.23 & 0.004 \\
\hline 19.8875 & 5 & 1 & 44.6532 & 4 & 4 & 24.7656 & 3 & 2 & 0.33 & 10.42 & $510^{-5}$ \\
\hline 18.9932 & 5 & 1 & 48.9931 & 4 & 5 & 29.9997 & 2 & 3 & 0.33 & 9.30 & 0.005 \\
\hline 17.1999 & 5 & 1 & 33.883 & 3 & 5 & 16.6832 & 3 & 2 & 0.33 & 6.40 & 0.002 \\
\hline 16.4713 & 3 & 3 & 23.0467 & 2 & 5 & 6.57529 & 1 & 1 & 0.33 & 4.36 & 0.004 \\
\hline 15.7683 & 4 & 1 & 41.7693 & 1 & 5 & 26.0007 & 1 & 3 & 0.33 & 8.17 & 0.005 \\
\hline
\end{tabular}

For this purpose we should use the properties of the symmetric matrix $S_{i j}^{m n}(11)$ possessing three real eigenvalues $\Omega_{i m n}(i=1,2,3)$ which are in the correspondence with three mutually orthogonal eigenvectors $L_{i m n}$.

We search for values $\Omega_{1 m_{1} n_{1}}, \Omega_{2 m_{2} n_{2}}$, and $\Omega_{3 m_{3} n_{3}}$ corresponding to the fixed shell's parameters $\sigma$, $\beta_{1}$ and $\beta_{2}$, which could satisfy the additive combinational resonance $\Omega_{2}=\Omega_{1}+\Omega_{3}$ (here subindices $m_{i} n_{i}$ are omitted for the ease of presentation), resulting in coupling of these particular three modes of vibration. Some results are shown in Table 1 , from which it is evident that the situation of such a combinational resonance could be realized rather often in real shells used as parts of different civil engineering structures.

\subsection{Numerical solution of general multi-term linear equations}

Using the numerical method proposed in [25][28], the procedure based on the reduction of the problem to a set of fractional differential equations to estimate numerically the solution of Eqs. (8-10) is as follows: let

$$
\begin{gathered}
Y_{1}=X_{1}, \\
Y_{2}=D^{\gamma} X_{1}=D^{\gamma} Y_{1}, \\
Y_{3}=D X_{1}=D Y_{1}, \\
\ddot{X}_{1}=D D X_{1}=D Y_{3} .
\end{gathered}
$$

First substitute these equalities in equation (8), resulting in

$$
\begin{gathered}
D Y_{3}=-\sum_{i=1}^{3} F_{1 m n} L_{i m n}^{I}-a_{1} Y_{2}-\Omega_{1}^{2} Y_{1}, \\
Y_{4}=X_{2} \\
Y_{5}=D^{\gamma} X_{2}=D^{\gamma} Y_{4}, \\
Y_{6}=D X_{2}=D Y_{4}, \\
\ddot{X}_{2}=D D X_{2}=D Y_{6}
\end{gathered}
$$

then in equation (9), resulting in

$$
\begin{gathered}
D Y_{6}=-\sum_{i=1}^{3} F_{2 m n} L_{i m n}^{I I}-a_{2} Y_{5}-\Omega_{2}^{2} Y_{4}, \\
Y_{7}=X_{3} \\
Y_{8}=D^{\gamma} X_{3}=D^{\gamma} Y_{7}, \\
Y_{9}=D X_{3}=D Y_{7}
\end{gathered}
$$




$$
\ddot{X}_{3}=D D X_{3}=D Y_{9},
$$

$$
D Y_{9}=-\sum_{i=1}^{3} F_{3 m n} L_{i m n}^{I I}-a_{3} Y_{8}-\Omega_{3}^{2} Y_{7}
$$

Thus, the governing set of nine equations in nine unknown values $Y_{i}$ in the matrix form could be written as

$$
\begin{aligned}
& \left.\mid \begin{array}{rrrrrrrrr}
D^{\gamma} & 0 & 0 & 0 & 0 & 0 & 0 & 0 & 0 \\
D & 0 & 0 & 0 & 0 & 0 & 0 & 0 & 0 \\
0 & 0 & D & 0 & 0 & 0 & 0 & 0 & 0 \\
0 & 0 & 0 & D^{\gamma} & 0 & 0 & 0 & 0 & 0 \\
0 & 0 & 0 & D & 0 & 0 & 0 & 0 & 0 \\
0 & 0 & 0 & 0 & 0 & D & 0 & 0 & 0 \\
0 & 0 & 0 & 0 & 0 & 0 & D^{\gamma} & 0 & 0 \\
0 & 0 & 0 & 0 & 0 & 0 & D & 0 & 0 \\
0 & 0 & 0 & 0 & 0 & 0 & 0 & 0 & D
\end{array}\right] *\left[\begin{array}{c}
Y_{1} \\
Y_{2} \\
Y_{3} \\
Y_{4} \\
Y_{5} \\
Y_{6} \\
Y_{7} \\
Y_{8} \\
Y_{9}
\end{array}\right]= \\
& {\left[\begin{array}{ccc}
Y_{2} \\
Y_{3} \\
-\sum_{i=1}^{3} F_{1 m n} L_{i m n}^{I}-a_{1} Y_{2}-\Omega_{1}^{2} Y_{1} \\
Y_{5} \\
-\sum_{i=1}^{3} F_{2 m n} L_{i m n}^{I I}-a_{2} Y_{5}-\Omega_{2}^{2} Y_{4} \\
Y_{8} \\
\sum_{i=1}^{3} F_{3 m n} L_{i m n}^{I I I}-a_{3} Y_{8}-\Omega_{3}^{2} Y_{7}
\end{array}\right]}
\end{aligned}
$$

Two different types of discretization of derivatives in (25) could be utilized [25-28]. For the first order of differentiation, the trapezoidal rule is usually used:

$$
D Y=f \stackrel{\text { yields }}{\rightarrow} Y_{i}=Y_{i-1}+\frac{1}{2} h\left(f_{i}+f_{i-1}\right)
$$

So in our problem, the discrete derivatives (D) so we have will take the form

where

Let and finally in equation (10), resulting in

$$
Y_{i}-\frac{1}{2} h f_{i}=Y_{i-1}+\frac{1}{2} h\left(f_{i-1}\right) .
$$

To discretize the fractional derivative, the Diethelm's method could be used [25]:

$$
D^{\gamma} Y=\frac{1}{{ }^{\gamma} \chi_{i}}\left(\sum_{k=0}^{i}{ }^{\gamma} \omega_{k, i} Y_{i-k}+\frac{Y_{0}}{\alpha}\right)
$$

$$
{ }^{\gamma} \chi_{i}=(i h)^{\alpha} \Gamma(-\gamma)
$$

and ${ }^{\gamma} \omega_{k, 0}, \ldots,{ }^{\gamma} \omega_{k, i}[25,26]$ are the convolution weights derived from the fact that the fractional operator defined in terms of a convolution integral. We will use the weights of the quadrature formula [25]

$$
\begin{aligned}
& f(\gamma)=\gamma(1-\gamma) j^{-\gamma \gamma} \omega_{k, 0}= \\
& = \begin{cases}-1 & \text { for } k=0 \\
2 k^{1-\gamma}-(k-1)^{1-\gamma}-(k+1)^{1-\gamma} & \text { for } k=1,2, ., j-1 \\
(\gamma-1) k^{-\gamma}-(k-1)^{1-\gamma}+k^{1-\gamma} & \text { for } k=j\end{cases}
\end{aligned}
$$

Discretization of the equation

$$
D^{\gamma} Y_{1}=Y_{2}
$$

results in the following relationships (note $\alpha=\gamma$ ):

$$
\begin{gathered}
\frac{1}{\gamma} \chi_{i}\left(\sum_{k=0}^{i} \gamma \omega_{k, i} Y_{1 i-k}+\frac{Y_{10}}{\gamma}\right)=Y_{2} ; \\
\left({ }^{\gamma} \omega_{0, i} Y_{1 i}+\sum_{k=1}^{i}{ }^{\gamma} \omega_{k, i} Y_{1 i-k}+\frac{Y_{10}}{\alpha}\right)={ }^{\gamma} \chi_{i} Y_{2 i} ;
\end{gathered}
$$

$$
\sum_{k=1}^{i} \gamma \omega_{k, i} Y_{1 i-k}+\frac{Y_{10}}{\gamma}=S_{1 i-1},
$$


Numerical Analysis of Non-linear Vibrations of a Fractionally Damped Cylindrical Shell under the Additive Combinational Internal Resonance

$$
\begin{gathered}
\left({ }^{\gamma} \omega_{0, i} Y_{1 i}+s_{1 i-1}\right)={ }^{\gamma} \chi_{i} Y_{2 i}, \\
s_{1 i}=-{ }^{\gamma} \omega_{0, i} Y_{1 i}+{ }^{\gamma} \chi_{i} Y_{2 i} .
\end{gathered}
$$

By the trapezoidal rule we can represent

$$
Y_{3}=\mathrm{D} Y_{1}
$$

in a discrete form as

$$
Y_{1 i}=Y_{1 i-1}+\frac{h}{2}\left(Y_{3 i}+Y_{3 i-1}\right)
$$

So rearranging the terms

$$
Y_{1 i}-\frac{h}{2} Y_{3 i}=Y_{1 i-1}+\frac{h}{2} Y_{3 i-1}=s_{2 i-1}
$$

and utilizing the trapezoidal rule, we can discretize

$$
\begin{aligned}
& D Y_{3}=-\sum_{j=1}^{3} F_{1 m n} L_{j m n}^{I}-a_{1} Y_{2}-\Omega_{1}^{2} Y_{1} ; \\
& {\left[\begin{array}{ccccccccc}
-{ }^{\gamma} \omega_{0, i} & { }^{\gamma} \chi_{i} & 0 & 0 & 0 & 0 & 0 & 0 & 0 \\
1 & 0 & -\frac{h}{2} & 0 & 0 & 0 & 0 & 0 & 0 \\
\frac{h}{2} \Omega_{1}^{2} & \frac{h}{2} \alpha_{1} & 1 & 0 & 0 & 0 & 0 & 0 & 0 \\
0 & 0 & 0 & -{ }^{\gamma} \omega_{0, i} & { }^{\gamma} \chi_{i} & 0 & 0 & 0 & 0 \\
0 & 0 & 0 & 1 & 0 & -\frac{h}{2} & 0 & 0 & 0 \\
0 & 0 & 0 & \frac{h}{2} \Omega_{2}^{2} & \frac{h}{2} \alpha{ }_{2} & 1 & 0 & 0 & 0 \\
0 & 0 & 0 & 0 & 0 & 0 & -{ }^{\gamma} \omega & { }^{\gamma} \chi_{i} & 0 \\
0 & 0 & 0 & 0 & 0 & 0 & 1 & 0 & -\frac{h}{2} \\
0 & 0 & 0 & 0 & 0 & 0 & \frac{h}{2} \Omega_{3}^{2} & \frac{h}{2} \alpha_{3} & 1
\end{array}\right] *\left[\begin{array}{c}
Y_{1 i} \\
Y_{2 i} \\
Y_{3 i} \\
Y_{4 i} \\
Y_{5 i} \\
Y_{6 i} \\
Y_{7 i} \\
Y_{8 i} \\
Y_{9 i}
\end{array}\right]=\left[\begin{array}{c}
s_{1 i-1} \\
s_{2 i-1} \\
s_{3 i-1} \\
s_{4 i-1} \\
s_{5 i-1} \\
s_{6 i-1} \\
s_{7 i-1} \\
s_{8 i-1} \\
s_{9 i-1}
\end{array}\right]}
\end{aligned}
$$

Figure 1. Formula (40). 


\subsection{Numerical solution of the governing equations for the combinational additive internal resonance using the Runge- Kutta fourth order method}

To utilize the Runge-Kutta fourth order method to estimate numerically the solution of equations (15)-(20), we first rewrite these equations as follows:

$$
\begin{gathered}
\dot{a}_{1}=\frac{1}{2}\left(-\Omega_{1}^{-1} a_{23}^{I} a_{2} a_{3} \sin \delta-s_{1} a_{1}\right) ; \\
\dot{a}_{2}=\frac{1}{2}\left(\Omega_{2}^{-1} a_{13}^{I I} a_{1} a_{3} \sin \delta-s_{2} a_{2}\right) ; \\
\dot{a}_{3}=\frac{1}{2}\left(-\Omega_{3}^{-1} a_{12}^{I I I} a_{1} a_{2} \sin \delta-s_{3} a_{3}\right) ; \\
\dot{\varphi}_{1}=\frac{1}{2} \sigma_{1}+\frac{1}{2} \frac{a_{23}^{I}}{\Omega_{1}} \frac{a_{2} a_{3}}{a_{1}} \cos \delta ; \\
\dot{\varphi}_{2}=\frac{1}{2} \sigma_{2}+\frac{1}{2} \frac{a_{13}^{I I}}{\Omega_{2}} \frac{a_{1} a_{3}}{a_{2}} \cos \delta ; \\
\dot{\varphi}_{3}=\frac{1}{2} \sigma_{3}+\frac{1}{2} \frac{a_{12}^{I I I}}{\Omega_{3}} \frac{a_{1} a_{2}}{a_{3}} \cos \delta .
\end{gathered}
$$

\section{NUMERICAL RESULTS}

\subsection{Method 1: multi-step fractional differential equations.}

The numerical solution using the multi step method of equation (40) has been carried out at the dimensionless parameters presented in Table 1 (for the case presented in the first line), and the results are presented in Fig. 1 for different magnitudes of the fractional parameter.

\subsection{Method 2: the analysis of the amplitudes and phases using multiple time scales}

Variation of the fractional parameter $\gamma$ from 0 to 1 allows one to investigate vibrations of cylindrical shells in surrounding media with different viscous properties, including the pure elastic case at $\gamma=0$ and conventional KelvinVoigt model at $\gamma \rightarrow 1$.
The dynamic behavior of a cylindrical shell in a viscous medium at $\gamma=0.02,025,0.5$, and 0.98 , which is found by using the first and second methods, is shown in Figures 2 and 3, respectively, for the parameters taken from Table 1, which correspond to the combinational internal resonance at $\Omega_{2}=\Omega_{1}+\Omega_{3}$.

The behavior of amplitudes of vibrations reveals the exchange of energy between the generalized displacements of the system under the considered case of the combinational internal resonance.

\section{CONCLUSION}

Free damped vibrations of a shallow nonlinear thin cylindrical shell in a fractional derivative viscoelastic medium are investigated numerically by two different methods based on the new approach proposed in [20-23].

The numerical solutions of the damped vibrations of the nonlinear cylindrical shell subjected to the conditions of the internal resonance have been estimated, and good agreement between the two methods has been achieved. Within the first method, the generalized displacements of a coupled set of nonlinear ordinary differential equations of the second order are calculated using the numerical solution of nonlinear multi-term fractional differential equations by the procedure based on the reducing the problem to a system of fractional differential equations. According to the second method, the amplitudes and phases of nonlinear vibrations are estimated from the governing nonlinear differential equations describing amplitude-and-phase modulations for the case of the combinational internal resonance.

It has been shown that, as in [22], the phenomenon of the internal resonance could be very critical, since in a circular cylindrical shell the internal additive and difference combinational resonances are always present. The effect of viscosity on the energy exchange mechanism is analyzed. 
Numerical Analysis of Non-linear Vibrations of a Fractionally Damped Cylindrical Shell under the Additive Combinational Internal Resonance

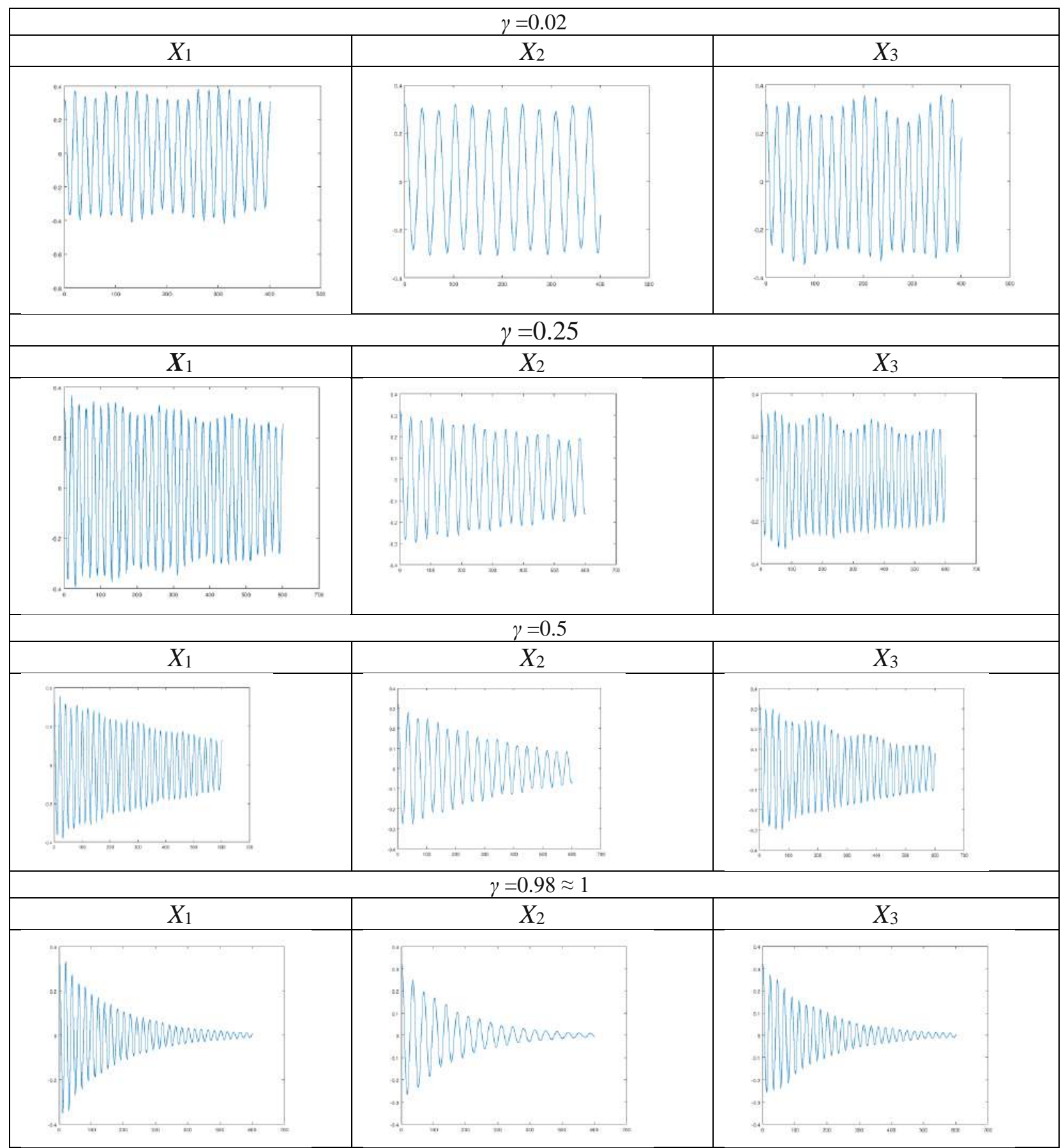

Figure 2. The time-dependence of the generalized displacements at different magnitudes of the fractional parameter.

\section{FUNDING}

This research was made possible by Grant No. 9.5138.2017/8.9 as a Government task from the Ministry of Education and Science of the Russian Federation.

Volume 14, Issue 4, 2018

\section{REFERENCES}

1. Rossikhin Yu.A., Shitikova M.V. SolitonLike Solutions in the Problems of Vibrations of Nonlinear Mechanical Systems: Survey // Nonlinear Systems Modeling, Estimation, and Stability, 2018, Chapter 4, pp. 65-87. 


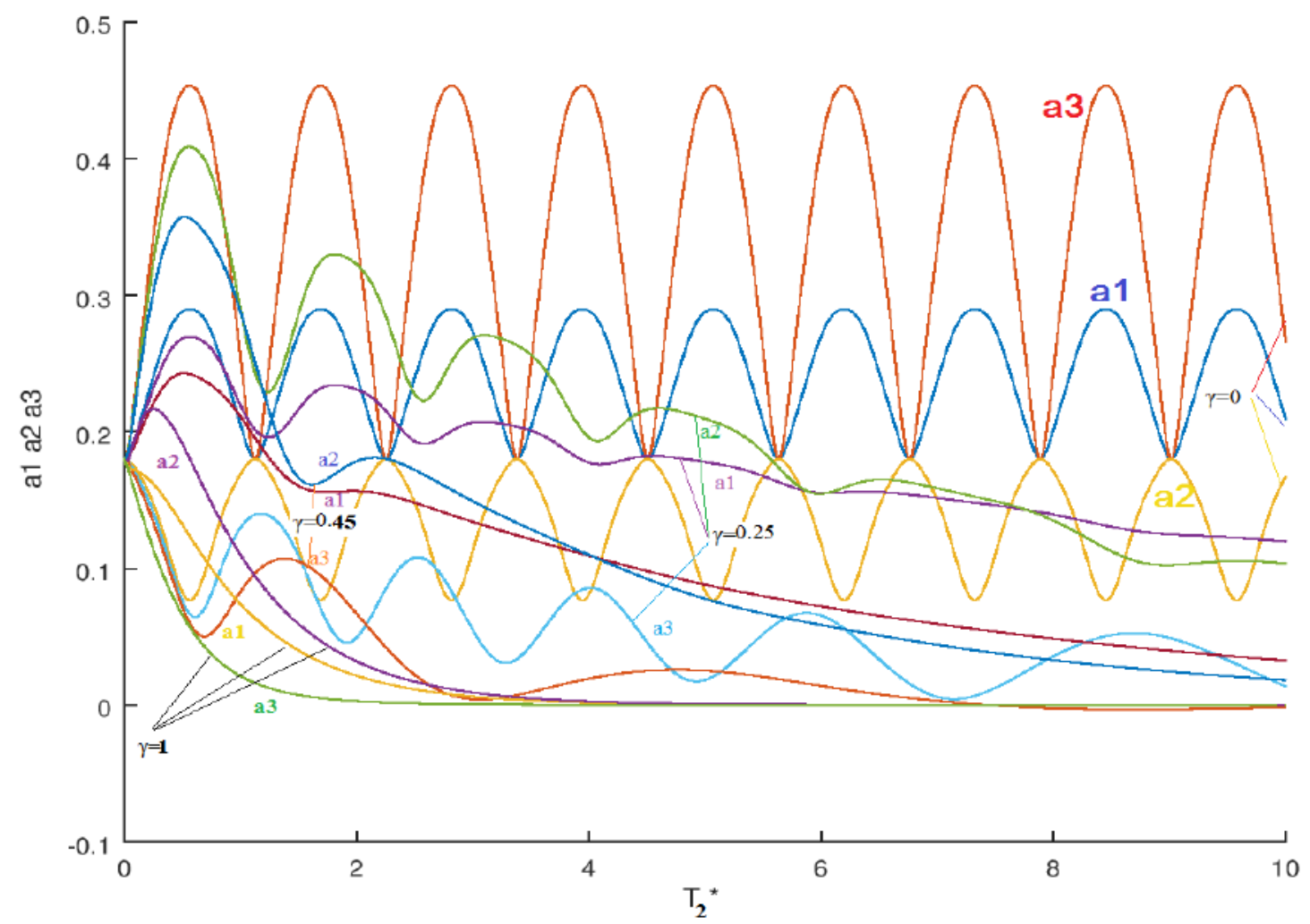

Figure 3. Dimensionless amplitude vs. dimensionless time as the solution of equations (41)-(46).

2. Nayfeh A.H., Balachandran S. Modal Interactions in Dynamical and Structural Systems. // Applied Mechanics Reviews, 1989, Vol. 42, pp. S175-S201.

3. Nayfeh A.H. Nonlinear Interaction: Analytical, Computational, and Experimental Methods. New York: Wiley, 2000.

4. Popov A.A., Thompson J.M.T., McRobie F.A. Low Dimensional Models of Shell Vibrations. Parametrically Excited Vibrations of Cylindrical Shells. // Journal of Sound and Vibration, 1998, Vol. 209, pp. 163-186.

5. Amabili M., Paidoussis M. Review of Studies on Geometrically Nonlinear Vibrations and Dynamics of Circular Cylindrical Shells and Panels, with and Without Fluid - Structure Interaction. // Applied Mechanics Reviews, 2003, Vol. 56, No. 4, pp. 349-381.

6. Nayfeh A.H., Raouf R.A. Non-Linear Oscillation of Circular Cylindrical Shells. // International Journal of Solids and Structures, 1987, Vol. 23, pp. 1625-1638.
7. McRobie F.A., Popov A.A., Thompson J.M.T. Auto-Parametric Resonance in Cylindrical Shells Using Geometric Averaging. // Journal of Sound and Vibration, 1999, Vol. 227, pp. 65-84.

8. Avramov K.V. Nonlinear Forced Vibrations of a Cylindrical Shell with Two Internal Resonances. // International Applied Mechanics, 2006, Vol. 42, No. 2, pp. 169-175.

9. Popov A.A. Auto-Parametric Resonance in Thin Cylindrical Shells Using the Slow Fluctuation. // Thin-Walled Structures, 2004, Vol. 42, pp. 475-495.

10. Breslavsky I.D., Amabili M. Nonlinear Vibrations of a Circular Cylindrical Shell with Multiple Internal Resonances Under Multi-Harmonic Excitation. // Nonlinear Dynamics, 2018, Vol. 93, pp. 53-62

11. Cheshko K.F., Polishchuk O.F., Avramov K.V. Eksperimental'nyj i Chislennyj Analiz Svobodnyh Kolebanij Pologoj Obolochki [Experimental and numerical analysis of free vibrations of a 
Numerical Analysis of Non-linear Vibrations of a Fractionally Damped Cylindrical Shell under the Additive Combinational Internal Resonance

shallow shell]. // Visnik KhTU. Series: Dynamika i Moshchnost Mashin, 2017, No. 40, pp. 81-85.

12. Rossikhin Yu.A., Shitikova M.V. Free Non-Linear Vibrations of an Elastic Cylindrical Shell Under the Conditions of the Internal Resonance. // Proceedings of the 18th International Congress on Sound and Vibration, July 11-14, 2011, Rio de Janeiro, Brazil, 2011, Vol. 4, pp. 2775-2782.

13. Rodrigues L., Gonçalves P.B., Silva F.M.A. Internal Resonances in a Transversally Excited Imperfect Circular Cylindrical Shell. // Procedia Engineering, 2017, Vol. 199, pp. 838-843.

14. Kubenko V.D., Koval'chuk P.S. Nonlinear Problems of the Vibration of Thin Shell (Review). // International Applied Mechanics, 1998, Vol. 34, No. 8, pp. 703-728.

15. Lee Y.S. Review on the Cylindrical Shell Research. // Transactions of the Korean Society of Mechanical Engineers, 2009, Vol. 33(1), pp. 1-26.

16. Alijani F., Amabili $M$. Non-Linear Vibrations of Shells: A literature Review from 2003 to 2013. // International Journal of Non-Linear Mechanics, 2014, Vol. 58, pp. 233-257.

17. Rossikhin Yu.A., Shitikova M.V. Application of Fractional Calculus for Analysis of Nonlinear Damped Vibrations of Suspension Bridges. // ASCE Journal of Engineering Mechanics, 1998, Vol. 124, pp. 1029-1036.

18. Rossikhin Yu.A, Shitikova M.V. Application of Fractional Calculus for Dynamic Problems of Solid Mechanics: Novel Trends and Recent Results // Applied Mechanics Reviews, 2010, Vol. 63(1), Article ID 010801, 52 pages.

19. Rossikhin Yu.A. Reflections on Two Parallel Ways in Progress of Fractional Calculus in Mechanics of Solids // Applied Mechanics Reviews, 2010, Vol. 63(1), Article ID 01071, 12 pages.
20. Rossikhin Yu.A., Shitikova M.V. Analysis of Damped Vibrations of a Cylindrical Shell Embedded into a Fractional Derivative Viscoelastic Medium. // Vibration Problems ICOVP 2011, Supplement, Proceedings of the 10th International Conference on Vibration Problems, Sept.5-8, 2011, Prague, Czech Republic, 2011, pp. 485-490.

21. Shitikova M.V., Rossikhin Yu.A. A New Approach for Studying Nonlinear Dynamic Response of a Thin Fractionally Damped Cylindrical Shell with Internal Resonances of the Order of $\varepsilon$. // "Shell and Membrane Theories in Mechanics and Biology: From Macro- to Nanoscale Structures" (H. Altenbach and G.I Mikhasev, Eds) Advanced Structured Materials, 2015, Vol. 45, Chapter 17, pp. 301-321.

22. Rossikhin Yu.A., Shitikova M.V. Analysis of Non-Linear Vibrations of a Fractionally Damped Cylindrical Shell Under the Conditions of Combinational Internal Resonance. // Computational Problems in Science and Engineering / Ed. by Mastorakis N., Bulucea A., Tsekouras G. Springer, 2015, Lecture Notes in Electrical Engineering, Vol. 343, Chapter 3, pp. 59-107.

23. Rossikhin Yu.A., Shitikova M.V. Nonlinear Dynamic Response of a Fractionally Damped Cylindrical Shell with a Three-to-One Internal Resonance. // Applied Mathematics and Computation, 2015, Vol. 257, pp. 498-525.

24. Rossikhin Y.A., Shitikova M.V., Ajarmah B. Numerical Analysis of NonLinear Vibrations of a Fractionally Damped Cylindrical Shell Under the Conditions of Combinational Internal Resonance. // MATEC Web of Conferences, 2018, Vol. 148, Paper ID 030062017.

25. Diethelm K. An Algorithm for the Numerical Solution of Differential Equations of Fractional Order. // Electronic Transactions on Numerical Analysis, 1997, Vol. 5, pp. 1-6. 
26. Diethelm K. Numerical Approximation of Fnite-Part Integrals with Generalised Compound Quadrature Formulae. // Hildesheimer Informatikberichte, 1995.

27. Edwards J.T., Simpson A.C. The Numerical Solution of Linear Multi-Term Fractional Differential Equations: Systems of Equations. // Journal of Computational and Applied Mathematics, 2002, Vol. 148, pp. 401-418.

28. Baleanu D., Diethelm K., Scalas E., Trujillo J.J. Fractional Calculus: Models and Numerical Methods. Singapore: World Scientific, 2012.

29. Rossikhin Yu.A., Shitikova M.V. Nonlinear Free Damped Vibrations of Suspension Bridges with Uncertain Fractional Damping. // Journal Europeen des Systemes Automatises, 2008, Vol. 42, No. 6-8, pp. 879-894.

30. Samko S.G., Kilbas A.A., Marichev O.I. Integraly i proizvodnye drobnogo poryadka i nekotorye ih prilozheniya [Fractional Integrals and Derivatives. Theory and Applications]. Minsk, Nauka i Tekhnika, Minsk, 1988 Engl. transl. by Gordon and Breach Science Publ., Amsterdam 1993.

31. Emama S.A., Nayfeh A.H. Non-Linear Response of Buckled Beams to 1:1 and 3:1 Internal Resonances. // Int. J. Non-Linear Mech., 2013, Vol. 52, pp. 12-25.

\section{СПИСОК ЛИТЕРАТУРЫ}

1. Rossikhin Yu.A., Shitikova M.V. SolitonLike Solutions in the Problems of Vibrations of Nonlinear Mechanical Systems: Survey // Nonlinear Systems Modeling, Estimation, and Stability, 2018, Chapter 4, pp. 65-87.

2. Nayfeh A.H., Balachandran S. Modal Interactions in Dynamical and Structural Systems. // Applied Mechanics Reviews, 1989, Vol. 42, pp. S175-S201.

3. Nayfeh A.H. Nonlinear Interaction: Analytical, Computational, and
Experimental Methods. New York: Wiley, 2000.

4. Popov A.A., Thompson J.M.T., McRobie F.A. Low Dimensional Models of Shell Vibrations. Parametrically Excited Vibrations of Cylindrical Shells. // Journal of Sound and Vibration, 1998, Vol. 209, pp. 163-186.

5. Amabili M., Paidoussis M. Review of Studies on Geometrically Nonlinear Vibrations and Dynamics of Circular Cylindrical Shells and Panels, with and Without Fluid - Structure Interaction. // Applied Mechanics Reviews, 2003, Vol. 56, No. 4, pp. 349-381.

6. Nayfeh A.H., Raouf R.A. Non-Linear Oscillation of Circular Cylindrical Shells. // International Journal of Solids and Structures, 1987, Vol. 23, pp. 1625-1638.

7. McRobie F.A., Popov A.A., Thompson J.M.T. Auto-Parametric Resonance in Cylindrical Shells Using Geometric Averaging. // Journal of Sound and Vibration, 1999, Vol. 227, pp. 65-84.

8. Avramov K.V. Nonlinear Forced Vibrations of a Cylindrical Shell with Two Internal Resonances. // International Applied Mechanics, 2006, Vol. 42, No. 2, pp. 169-175.

9. Popov A.A. Auto-Parametric Resonance in Thin Cylindrical Shells Using the Slow Fluctuation. // Thin-Walled Structures, 2004, Vol. 42, pp. 475-495.

10. Breslavsky I.D., Amabili M. Nonlinear Vibrations of a Circular Cylindrical Shell with Multiple Internal Resonances Under Multi-Harmonic Excitation. // Nonlinear Dynamics, 2018, Vol. 93, pp. 53-62

11. Чешко К.Ф., Полищук О.Ф., Аврамов К.В. Экспериментальный и численный анализ свободных колебаний пологой оболочки. // Вестник НТУ «ХПІ». Серия: Динамика и прочность машин, 2017, №40(1262), с. 81-85.

12. Rossikhin Yu.A., Shitikova M.V. Free Non-Linear Vibrations of an Elastic Cylindrical Shell Under the Conditions of the Internal Resonance. // Proceedings of the 
Numerical Analysis of Non-linear Vibrations of a Fractionally Damped Cylindrical Shell under the Additive Combinational Internal Resonance

18th International Congress on Sound and Vibration, July 11-14, 2011, Rio de Janeiro, Brazil, 2011, Vol. 4, pp. 2775-2782.

13. Rodrigues L., Gonçalves P.B., Silva F.M.A. Internal Resonances in a Transversally Excited Imperfect Circular Cylindrical Shell. // Procedia Engineering, 2017, Vol. 199, pp. 838-843.

14. Kubenko V.D., Koval'chuk P.S. Nonlinear Problems of the Vibration of Thin Shell (Review). // International Applied Mechanics, 1998, Vol. 34, No. 8, pp. 703-728.

15. Lee Y.S. Review on the Cylindrical Shell Research. // Transactions of the Korean Society of Mechanical Engineers, 2009, Vol. 33(1), pp. 1-26.

16. Alijani F., Amabili M. Non-Linear Vibrations of Shells: A literature Review from 2003 to 2013. // International Journal of Non-Linear Mechanics, 2014, Vol. 58, pp. 233-257.

17. Rossikhin Yu.A., Shitikova M.V. Application of Fractional Calculus for Analysis of Nonlinear Damped Vibrations of Suspension Bridges. // ASCE Journal of Engineering Mechanics, 1998, Vol. 124, pp. 1029-1036.

18. Rossikhin Yu.A, Shitikova M.V. Application of Fractional Calculus for Dynamic Problems of Solid Mechanics: Novel Trends and Recent Results // Applied Mechanics Reviews, 2010, Vol. 63(1), Article ID 010801, 52 pages.

19. Rossikhin Yu.A. Reflections on Two Parallel Ways in Progress of Fractional Calculus in Mechanics of Solids // Applied Mechanics Reviews, 2010, Vol. 63(1), Article ID 01071, 12 pages.

20. Rossikhin Yu.A., Shitikova M.V. Analysis of Damped Vibrations of a Cylindrical Shell Embedded into a Fractional Derivative Viscoelastic Medium. // Vibration Problems ICOVP 2011, Supplement, Proceedings of the 10th International Conference on Vibration
Problems, Sept.5-8, 2011, Prague, Czech Republic, 2011, pp. 485-490.

21. Shitikova M.V., Rossikhin Yu.A. A New Approach for Studying Nonlinear Dynamic Response of a Thin Fractionally Damped Cylindrical Shell with Internal Resonances of the Order of $\varepsilon$. // "Shell and Membrane Theories in Mechanics and Biology: From Macro- to Nanoscale Structures" (H. Altenbach and G.I Mikhasev, Eds) Advanced Structured Materials, 2015, Vol. 45, Chapter 17, pp. 301-321.

22. Rossikhin Yu.A., Shitikova M.V. Analysis of Non-Linear Vibrations of a Fractionally Damped Cylindrical Shell Under the Conditions of Combinational Internal Resonance. // Computational Problems in Science and Engineering / Ed. by Mastorakis N., Bulucea A., Tsekouras G. Springer, 2015, Lecture Notes in Electrical Engineering, Vol. 343, Chapter 3, pp. 59-107.

23. Rossikhin Yu.A., Shitikova M.V. Nonlinear Dynamic Response of a Fractionally Damped Cylindrical Shell with a Three-to-One Internal Resonance. // Applied Mathematics and Computation, 2015, Vol. 257, pp. 498-525.

24. Rossikhin Y.A., Shitikova M.V., Ajarmah B. Numerical Analysis of NonLinear Vibrations of a Fractionally Damped Cylindrical Shell Under the Conditions of Combinational Internal Resonance. // MATEC Web of Conferences, 2018, Vol. 148, Paper ID 030062017.

25. Diethelm K. An Algorithm for the Numerical Solution of Differential Equations of Fractional Order. // Electronic Transactions on Numerical Analysis, 1997, Vol. 5, pp. 1-6.

26. Diethelm K. Numerical Approximation of Fnite-Part Integrals with Generalised Compound Quadrature Formulae. // Hildesheimer Informatikberichte, 1995.

27. Edwards J.T., Simpson A.C. The Numerical Solution of Linear Multi-Term Fractional Differential Equations: Systems of Equations. // Journal of Computational 
and Applied Mathematics, 2002, Vol. 148, pp. 401-418.

28. Baleanu D., Diethelm K., Scalas E., Trujillo J.J. Fractional Calculus: Models and Numerical Methods. Singapore: World Scientific, 2012.

29. Rossikhin Yu.A., Shitikova M.V. Nonlinear Free Damped Vibrations of Suspension Bridges with Uncertain Fractional Damping. // Journal Europeen des Systemes Automatises, 2008, Vol. 42, No. 6-8, pp. 879-894.

30. Самко С.Г., Килбас А.А., Маричев

О.И. Интегралы и производные дробного порядка и некоторые их приложения. - Минск: Наука и техника, 1987. -688 c.

31. Emama S.A., Nayfeh A.H. Non-Linear Response of Buckled Beams to 1:1 and 3:1 Internal Resonances. // Int. J. Non-Linear Mech., 2013, Vol. 52, pp. 12-25.

Marina V. Shitikova, Advisor of the Russian Academy of Architecture and Construction Sciences, Prof., Dr.Sc., Research Center on Dynamics of Solids and Structures; Voronezh State Technical University; 84, 20 let Oktyabrya, Voronezh, 394006, Russia;

phone +7 (473) 271-52-68; fax +7 (473) 271-52-68;

E-mail: mvs@vgasu.vrn.ru.

Basem Ajarmah, Al-istiqlal University, Jericho, Palestine, Opposite Hisham's palace; P.O.Box 10;

phone +972/022322194; fax: +972/022322197;

E-mail: basem@pass.ps.

Шитикова Марина Вячеславовна, советник РААСН, профессор, доктор физико-математических наук; профессор кафедры информационных технологий и автоматизированного проектирования в строительстве; Воронежский государственный технический университет; 394006, Россия, г. Воронеж, ул. 20 лет Октября, д. 84,

тел. +7 (473) 271-52-68; факс +7 (473) 271-52-68;

E-mail: mvs@vgasu.vrn.ru.

Басем Айармах, Al-istiqlal University, г. Иерихон, Палестина, Opposite Hisham's palace; P.O.Box 10; тел. +972/022322194; факс +972/022322197;

E-mail: basem@pass.ps. 